\title{
Pythagorean Triangle with Area/ Perimeter as a special polygonal number
}

\author{
M.A.Gopalan ${ }^{1}$, Manju Somanath ${ }^{2}$, K.Geetha ${ }^{3}$ \\ 1. Department of Mathematics, Shrimati Indira Gandhi College, Trichy. \\ 2. Department of Mathematics, National College, Trichy. \\ 3. Department of Mathematics, Cauvery College for Women, Trichy.
}

Abstract: Patterns of Pythagorean triangles, in each of which the ratio Area/ Perimeter is represented by some polygonal number. A few interesting relations among the sides are also given.

Keyword: Polygonal number, Pyramidal number, Centered polygonal number, Centered pyramidal number, Special number

\section{Introduction}

The method of obtaining three non-zero integers $\mathrm{x}, \mathrm{y}$ and $\mathrm{z}$ under certain conditions satisfying the relation $x^{2}+y^{2}=z^{2}$ has been a matter of interest to various Mathematicians [1, 3, 4, 5,6]. In [7-15], special Pythagorean problems are studied. In this communication, we present yet another interesting Pythagorean problem. That is, we search for patterns of Pythagorean triangles where, in each of which, the ratio Area/ Perimeter is represented by a special polygonal number. Also, a few relations among the sides are presented.

Notation

$p_{n}^{m}=$ Pyramidal number of rank $\mathrm{n}$ with sides $\mathrm{m}$

$t_{m, n}=$ Polygonal number of rank $\mathrm{n}$ with sides $\mathrm{m}$

$j a l_{n}=$ Jacobsthal Lucas number

$j a_{n}=$ Jacobsthal number

$c t_{m, n}=$ Centered Polygonal number of rank $\mathrm{n}$ with sides $\mathrm{m}$

$c p_{n}^{m}=$ Centered Pyramidal number of rank $\mathrm{n}$ with sides $\mathrm{m}$

$g_{n}=$ Gnomonic number of rank $\mathrm{n}$ with sides $\mathrm{m}$

$p_{n}=$ Pronic number

$\operatorname{carl}_{n}=$ Carol number

$k y_{n}=$ Kynea number

\section{Method of Analysis}

The most cited solution of the Pythagorean equation,

is represented by

$$
x^{2}+y^{2}=z^{2}
$$

$$
x=2 u v, y=u^{2}-v^{2}, z=u^{2}+v^{2}(u>v>0)
$$

Pattern 1:

Denoting the Area and Perimeter of the triangle by A and P respectively, the assumption

$$
\frac{A}{P}=t_{11, n}
$$

leads to the equation

$$
q(p-q)=n(9 n-7)
$$

This equation is equivalent to the following two systems I and II respectively: 


\begin{tabular}{|c|c|}
\hline$p-q$ & $Q$ \\
\hline $9 n-7$ & $N$ \\
\hline$N$ & $9 n-7$ \\
\hline
\end{tabular}

In what follows, we obtain the values of the generators $\mathrm{p}, \mathrm{q}$ and hence the corresponding sides of the Pythagorean triangle

\section{Case 1:}

On evaluation, the values of the generators satisfying system I are,

$$
p=10 n-7, q=n
$$

Employing (2), the sides of the corresponding, Pythagorean triangle are given by

$$
x(n)=20 n^{2}-14 n, y(n)=99 n^{2}-140 n+49, z(n)=101 n^{2}-140 n+49
$$

Examples:

\section{Properties:}

\begin{tabular}{|c|c|c|c|c|c|}
\hline $\mathbf{n}$ & $\mathbf{X}$ & $\mathbf{Y}$ & $\mathbf{Z}$ & $\mathbf{A}$ & $\mathbf{P}$ \\
\hline 1 & 6 & 8 & 10 & 24 & 24 \\
\hline 2 & 52 & 165 & 173 & 4290 & 390 \\
\hline 3 & 138 & 520 & 538 & 35880 & 1196 \\
\hline 4 & 264 & 1073 & 1105 & 141636 & 2442 \\
\hline 5 & 430 & 1824 & 1874 & 392160 & 4128 \\
\hline
\end{tabular}

1) $6\left\{\frac{(z-y) x-8 n^{3}}{t_{22, n}}\right\}$ is a Nasty number[2]

2) $10 x-y-z \equiv 42(\bmod 140)$

3) $\quad(10 x(2 n+1)-y(2 n+1)-z(2 n+1)+78)^{2}=140^{2}\left(8 t_{3, n+1}\right)$

Case 2:

On evaluation, the values, of the generators satisfying system II are

$$
p=10 n-7, q=9 n-7
$$

Using (2), the corresponding Pythagorean triangle is

$$
x(n)=180 n^{2}-266 n+98, y(n)=19 n^{2}-14 n, z(n)=181 n^{2}-266 n+98
$$

\section{Examples:}

\begin{tabular}{|c|c|c|c|c|c|}
\hline $\mathbf{n}$ & $\mathbf{X}$ & $\mathbf{Y}$ & $\mathbf{z}$ & $\mathbf{A}$ & $\mathbf{P}$ \\
\hline 1 & 12 & 5 & 13 & 30 & 30 \\
\hline 2 & 286 & 48 & 290 & 6864 & 624 \\
\hline 3 & 920 & 129 & 929 & 59340 & 1978 \\
\hline 4 & 1914 & 248 & 1930 & 1081 & 2372 \\
\hline 5 & 3268 & 405 & 3293 & 661770 & 6966 \\
\hline
\end{tabular}

\section{Properties:}

$$
\begin{aligned}
& \text { 1) } z-9 y-s_{n}-t_{10, n} \equiv 97(\bmod 131) \\
& \text { 2) } 10 y-z-x+5 t_{65, n}+p_{n}^{5} \equiv 98(\bmod 228) \\
& \text { 3) } 10 x-9(y+z) \equiv 98(\bmod 140)
\end{aligned}
$$

\section{Pattern 2:}

The assumption

$$
\frac{A}{P}=t_{12, n}
$$

leads to the equation

$$
q(p-q)=2 n(5 n-4)
$$

This equation is equivalent to the following two systems I and II respectively 


\begin{tabular}{|c|c|}
\hline$p-q$ & $Q$ \\
\hline $5 n-7$ & $2 n$ \\
\hline $2 n$ & $5 n-7$ \\
\hline
\end{tabular}

As in the previous case, we obtain the values of the generators $\mathrm{u}, \mathrm{v}$ and hence the corresponding sides of the Pythagorean triangle.

Case 1:

On evaluation, the values of the generators satisfying system I are,

$$
p=7 n-4, q=2 n
$$

Using (2), the corresponding Pythagorean triangle is

\section{Examples:}

$$
x(n)=28 n^{2}-16 n, y(n)=45 n^{2}-56 n+16, z(n)=53 n^{2}-56 n+16
$$

\begin{tabular}{|c|c|c|c|c|c|}
\hline $\mathbf{n}$ & $\mathbf{X}$ & $\mathbf{Y}$ & $\mathbf{z}$ & $\mathbf{A}$ & $\mathbf{P}$ \\
\hline 1 & 12 & 5 & 13 & 30 & 30 \\
\hline 2 & 80 & 84 & 116 & 3360 & 280 \\
\hline 3 & 204 & 253 & 325 & 25806 & 782 \\
\hline 4 & 384 & 512 & 640 & 98304 & 1536 \\
\hline 5 & 620 & 861 & 1061 & 266910 & 2542 \\
\hline
\end{tabular}

\section{Properties:}

1) $\quad x(z-y)-8 t_{58, n} \equiv 0(\bmod 88)$

2) $x+y-z-4 t_{12, n}=0$

3) $6\left\{\frac{y+8 n^{2}}{z}\right\}$ is a nasty number

\section{Case 2:}

On evaluation, the values of the generators satisfying the system II are

$$
p=7 n-4, q=6 n-4
$$

Using (2), the corresponding Pythagorean triangle is

\section{Examples:}

$$
x(n)=84 n^{2}-104 n+32, y(n)=13 n^{2}-8 n, z(n)=85 n^{2}-104 n+32
$$

\begin{tabular}{|c|c|c|c|c|c|}
\hline $\mathbf{n}$ & $\mathbf{x}$ & $\mathbf{Y}$ & $\mathbf{z}$ & $\mathbf{A}$ & $\mathbf{P}$ \\
\hline 1 & 12 & 5 & 13 & 30 & 30 \\
\hline 2 & 160 & 44 & 164 & 3520 & 368 \\
\hline 3 & 476 & 109 & 485 & 25942 & 1070 \\
\hline 4 & 960 & 200 & 976 & 96000 & 2136 \\
\hline 5 & 1612 & 317 & 1637 & 255502 & 3566 \\
\hline
\end{tabular}

\section{Properties:}

$$
\begin{aligned}
& \text { 1) }(z-x) y-2 c t_{13, n^{2}}+t_{44, n} \equiv-2(\bmod 20) \\
& \text { 2) } x-6 y+32 g_{n}-8 n \text { is a Nasty number } \\
& \text { 3) } x(n+1)-6 y(n+1)-12 t_{3, n+1} \equiv 30(\bmod 62)
\end{aligned}
$$

\section{Pattern 3:}

Under our assumption

$$
\frac{A}{P}=t_{13, n}
$$

leads to the equation

$$
q(p-q)=n(11 n-7)
$$

This equation is equivalent to the following two systems I and II, 


\begin{tabular}{|c|c|}
\hline$p-q$ & $Q$ \\
\hline $11 n-7$ & $N$ \\
\hline$n$ & $11 n-7$ \\
\hline
\end{tabular}

\section{Case 1:}

On evaluation, the values of the generators satisfying system I are

$$
p=12 n-7, q=n
$$

In view of (2) the corresponding Pythagorean triangle is

$x(n)=24 n^{2}-14 n, y(n)=143 n^{2}-168 n+49, z(n)=145 n^{2}-168 n+49$

Examples:

\section{Properties:}

\begin{tabular}{|c|c|c|c|c|c|}
\hline $\mathbf{n}$ & $\mathbf{x}$ & $\mathbf{Y}$ & $\mathbf{z}$ & $\mathbf{A}$ & $\mathbf{P}$ \\
\hline 1 & 10 & 24 & 26 & 120 & 60 \\
\hline 2 & 68 & 285 & 293 & 9690 & 646 \\
\hline 3 & 174 & 832 & 850 & 72384 & 1856 \\
\hline 4 & 328 & 1665 & 1697 & 273060 & 3690 \\
\hline 5 & 530 & 2784 & 2834 & 737760 & 6148 \\
\hline
\end{tabular}

1) $6\left\{\frac{y+2 n^{2}}{z}\right\}$ is a Nasty number

2) $z-6 x-2 t_{3, n} \equiv 49(\bmod 85)$

3) $\quad n(z-y)+x-6 p_{n}^{4}-2 t_{23, n} \equiv 0(\bmod 4)$

Case 2:

On evaluation, the values of the generators satisfying system II are

$$
p=11 n-7, q=10 n-7
$$

Employing (2), the corresponding Pythagorean triangle is

$$
x=220 n^{2}-294 n+98, y=21 n^{2}-14 n, z=221 n^{2}-294 n+98
$$

\section{Examples:}

\begin{tabular}{|c|c|c|c|c|c|}
\hline $\mathbf{n}$ & $\mathbf{x}$ & $\mathbf{Y}$ & $\mathbf{z}$ & $\mathbf{A}$ & $\mathbf{P}$ \\
\hline 1 & 24 & 7 & 84 & 56 & 25 \\
\hline 2 & 390 & 56 & 10920 & 840 & 394 \\
\hline 3 & 1196 & 147 & 87906 & 2548 & 1205 \\
\hline 4 & 2442 & 280 & 341880 & 5180 & 2458 \\
\hline 5 & 4128 & 455 & 939120 & 8736 & 4153 \\
\hline
\end{tabular}

\section{Properties:}

1) $x-10 y-t_{22, n} \equiv 98(\bmod 145)$

2) $n(z-10 y)-6 p_{n}^{13}+52 t_{8, n}+2 t_{3, n} \equiv 0(\bmod 3)$

3) $(z-x)(x-13 y)=2 t_{15, n^{2}}-61 c p_{n}^{6}+506 p_{n}^{5}$

\section{Pattern 4:}

Under the assumption

$$
\frac{A}{P}=t_{14, n}
$$

leads to the equation

$$
q(p-q)=2 n(6 n-5)
$$

This equation is equivalent to the following two systems

\begin{tabular}{|c|c|}
\hline$p-q$ & $Q$ \\
\hline $6 n-7$ & $2 n$ \\
\hline $2 n$ & $6 n-5$ \\
\hline
\end{tabular}


As in the previous case, we obtain the values of the generators $\mathrm{u}, \mathrm{v}$ and hence the corresponding sides of the Pythagorean triangle.

Case 1:

On evaluation, the values of generators satisfying system I are

$$
p=8 n-5, q=2 n
$$

Employing (2), the corresponding Pythagorean triangle is

$$
x=32 n^{2}-20 n, y=60 n^{2}-80 n+25, z=68 n^{2}-80 n+25
$$

Examples:

\begin{tabular}{|c|c|c|c|c|c|}
\hline $\mathbf{n}$ & $\mathbf{x}$ & $\mathbf{Y}$ & $\mathbf{z}$ & $\mathbf{A}$ & $\mathbf{P}$ \\
\hline 1 & 12 & 5 & 13 & 30 & 30 \\
\hline 2 & 88 & 105 & 137 & 4620 & 330 \\
\hline 3 & 228 & 325 & 397 & 37050 & 950 \\
\hline 4 & 432 & 665 & 793 & 143640 & 1890 \\
\hline 5 & 700 & 1125 & 1325 & 393750 & 3150 \\
\hline
\end{tabular}

Properties:

1) $z-2 x-t_{9, n} \equiv 25(\bmod 37)$

2) $\quad n(z-y)=8 c p_{n}^{6}$

3) $\quad n x-6 c p_{n}^{30}-2 p_{n}^{8}+t_{44, n} \equiv 0(\bmod 5)$

Case 2:

On evaluation, the values of generators satisfying II are

$$
p=8 n-5, q=6 n-5
$$

Employing (2), the corresponding Pythagorean triangle is

$$
x(n)=96 n^{2}-140 n+50, y(n)=28 n^{2}-20 n, z(n)=100 n^{2}-140 n+50
$$

\section{Examples:}

\begin{tabular}{|c|c|c|c|c|c|}
\hline $\mathbf{n}$ & $\mathbf{x}$ & $\mathbf{Y}$ & $\mathbf{z}$ & $\mathbf{A}$ & $\mathbf{P}$ \\
\hline 1 & 6 & 8 & 10 & 24 & 24 \\
\hline 2 & 154 & 72 & 170 & 5472 & 1216 \\
\hline 3 & 494 & 192 & 530 & 47424 & 1216 \\
\hline 4 & 1026 & 368 & 1090 & 188784 & 2484 \\
\hline 5 & 1750 & 600 & 1850 & 525000 & 4200 \\
\hline
\end{tabular}

Properties:

1) $\quad \frac{x-3 y}{2}+20 g_{n}-5$ is a Nasty number

2) $\quad \frac{z}{2}-c t_{23, n} \equiv 23(\bmod 116)$

3) $\frac{x}{2}+3 y-23=6 c t_{8, n}-2 g_{n}$

\section{Pattern 5:}

The assumption

$$
\frac{A}{P}=t_{15, n}
$$

leads to the equation

$$
q(p-q)=n(13 n-11)
$$

The above equation is equivalent to the following two systems I and II respectively 


\begin{tabular}{|c|c|}
\hline$p-q$ & $Q$ \\
\hline $13 n-11$ & $N$ \\
\hline$n$ & $13 n-11$ \\
\hline
\end{tabular}

As in the previous case, we obtain the values of the generators $\mathrm{u}, \mathrm{v}$ and hence the corresponding sides of the Pythagorean triangle.

Case 1:

On evaluation, the values of generators satisfying system I are

$$
p=14 n-11, q=n
$$

Employing (2), the corresponding Pythagorean triangle is

\section{Examples:}

$$
x(n)=28 n^{2}-22 n, y(n)=195 n^{2}-308 n+121, z(n)=197 n^{2}-308 n+121
$$

\section{Properties:}

\begin{tabular}{|c|c|c|c|c|c|}
\hline $\mathbf{n}$ & $\mathbf{x}$ & $\mathbf{Y}$ & $\mathbf{z}$ & $\mathbf{A}$ & $\mathbf{P}$ \\
\hline 1 & 6 & 8 & 10 & 24 & 24 \\
\hline 2 & 68 & 285 & 293 & 9690 & 646 \\
\hline 3 & 186 & 952 & 970 & 88536 & 2108 \\
\hline 4 & 360 & 2009 & 2041 & 361620 & 4410 \\
\hline 5 & 590 & 3456 & 3506 & 1019520 & 7552 \\
\hline
\end{tabular}

1) $z-7 x-42 c p_{n}^{28}+196 c p_{n}^{6}-121$ is a perfect square

2) $\left(\frac{z-y}{2}\right)^{2}$ is biquadratic integer

3) $\frac{n x}{2}-6 c p_{n}^{13}+3 t_{24, n}+3 n$ is cubic integer

Case 2:

On evaluation, the values of generators satisfying system II are

$$
p=14 n-11, q=13 n-11
$$

Employing (2), the corresponding Pythagorean triangle is

$$
x(n)=364 n^{2}-594 n+242, y(n)=27 n^{2}-22 n, z(n)=365 n^{2}-594 n+242
$$

\section{Examples:}

\begin{tabular}{|c|c|c|c|c|c|}
\hline $\mathbf{n}$ & $\mathbf{x}$ & $\mathbf{Y}$ & $\mathbf{z}$ & $\mathbf{A}$ & $\mathbf{P}$ \\
\hline 1 & 12 & 5 & 13 & 30 & 30 \\
\hline 2 & 510 & 64 & 514 & 16320 & 1088 \\
\hline 3 & 1736 & 177 & 1745 & 153636 & 3658 \\
\hline 4 & 3690 & 344 & 3706 & 634680 & 7740 \\
\hline 5 & 6372 & 565 & 6397 & 1800090 & 13334 \\
\hline
\end{tabular}

\section{Properties:}

1) $\quad(z-x)(x-13 y)=2 t_{15, n^{2}}-561 c p_{n}^{6}+506 p_{n}^{5}$

2) $\quad(z-x) y=n^{2} t_{56, n}+4 c p_{n}^{6}$

3) $14 y-z-2 t_{15, n} \equiv-242(\bmod 275)$

\section{Pattern 6:}

The assumption

$$
\frac{A}{P}=t_{16, n}
$$

leads to the equation

$$
q(p-q)=2 n(7 n-6)
$$

The above equation is equivalent to the following two systems I and II respectively 


\begin{tabular}{|c|c|}
\hline $\mathrm{p}-\mathrm{q}$ & $\mathrm{Q}$ \\
\hline $7 \mathrm{n}-6$ & $2 \mathrm{n}$ \\
\hline $2 \mathrm{n}$ & $7 \mathrm{n}-6$ \\
\hline
\end{tabular}

As in the previous case, we obtain the values of the generators $u, v$ and hence the corresponding sides of the Pythagorean triangle.

Case 1:

On evaluation, the values of generators satisfying system I are

$$
p=9 n-6, q=2 n
$$

Employing (2), the corresponding Pythagorean triangle is

\section{Examples:}

$$
x(n)=36 n^{2}-24 n, y(n)=77 n^{2}-108 n+36, z(n)=85 n^{2}-108 n+36
$$

\begin{tabular}{|c|c|c|c|c|c|}
\hline $\mathbf{n}$ & $\mathbf{x}$ & $\mathbf{y}$ & $\mathbf{z}$ & $\mathbf{A}$ & $\mathbf{P}$ \\
\hline 1 & 12 & 5 & 13 & 30 & 30 \\
\hline 2 & 96 & 128 & 160 & 6144 & 384 \\
\hline 3 & 252 & 405 & 477 & 51030 & 1134 \\
\hline 4 & 480 & 836 & 964 & 200640 & 2280 \\
\hline 5 & 780 & 1421 & 1621 & 554190 & 3822 \\
\hline
\end{tabular}

\section{Properties:}

1) $8 x-3 y-2 t_{58, n} \equiv-108(\bmod 186)$

2) $x-t_{58, n}+8 n^{2} \equiv 0(\bmod 3)$

3) $z-y-c t_{16, n} \equiv-1(\bmod 8)$

Case 2:

On evaluation the values of generators satisfying equation II are

$$
p=9 n-6, q=7 n-6
$$

Employing (2), the corresponding Pythagorean triangle is

\section{Examples:}

$$
x(n)=126 n^{2}-192 n+72, y(n)=32 n^{2}-24 n, z(n)=130 n^{2}-192 n+72
$$

\section{Properties:}

\begin{tabular}{|c|c|c|c|c|c|}
\hline $\mathbf{n}$ & $\mathbf{x}$ & $\mathbf{y}$ & $\mathbf{z}$ & $\mathbf{A}$ & $\mathbf{P}$ \\
\hline 1 & 6 & 8 & 10 & 24 & 24 \\
\hline 2 & 192 & 80 & 208 & 7680 & 480 \\
\hline 3 & 630 & 216 & 666 & 68040 & 1512 \\
\hline 4 & 1320 & 416 & 1384 & 274560 & 3120 \\
\hline 5 & 2262 & 680 & 2362 & 769080 & 5304 \\
\hline
\end{tabular}

1) $4 y-2 x+z-48 g_{n}+24 n$ is a Nasty number

2) $y(2 n+1)-x-c t_{4, n} \equiv-65(\bmod 294)$

3) $z(n-1)-x-c t_{8, n}+132 g_{n}=189$

\section{Pattern 7:}

The assumption

$$
\frac{A}{P}=t_{17, n}
$$

leads to the equation

$$
q(p-q)=n(15 n-13)
$$

The above equation is equivalent to the following two systems I and II respectively

\begin{tabular}{|c|c|}
\hline $\mathrm{p}-\mathrm{q}$ & $\mathrm{Q}$ \\
\hline $15 \mathrm{n}-13$ & $\mathrm{~N}$ \\
\hline $\mathrm{n}$ & $15 \mathrm{n}-13$ \\
\hline
\end{tabular}


As in the previous case, we obtain the values of the generators $\mathrm{u}, \mathrm{v}$ and hence the corresponding sides of the Pythagorean triangle.

Case 1:

On evaluation, the values of generators satisfying system I are

$$
p=16 n-13, q=n
$$

Employing (2), the corresponding Pythagorean triangle is

Examples:

$$
x(n)=32 n^{2}-26 n, y(n)=255 n^{2}+169-416 n, z(n)=257 n^{2}+169-416 n
$$

\begin{tabular}{|c|c|c|c|c|c|}
\hline $\mathbf{n}$ & $\mathbf{x}$ & $\mathbf{y}$ & $\mathbf{z}$ & $\mathbf{A}$ & $\mathbf{P}$ \\
\hline 1 & 6 & 8 & 10 & 24 & 24 \\
\hline 2 & 76 & 357 & 365 & 13566 & 798 \\
\hline 3 & 210 & 1216 & 1234 & 127680 & 2660 \\
\hline 4 & 408 & 2585 & 2617 & 527340 & 5610 \\
\hline 5 & 670 & 4464 & 4514 & 1495440 & 9648 \\
\hline
\end{tabular}

Properties:

1) $\quad x\left(2^{n}\right)-8 j a l_{2 n}+26 m e r_{n}+34=0$

2) $\quad(y-z)\left(2^{n}\right)-2=\operatorname{car} 1_{\mathrm{n}}+k y_{n}$

Case 2:

On evaluation the values of generators satisfying system II are given by

$$
p=16 n-13, q=15 n-13
$$

Employing (2), the corresponding Pythagorean triangle is

$x(n)=480 n^{2}-806 n+338, y(n)=31 n^{2}-26 n, \quad z(n)=481 n^{2}-806 n+338$

Examples:

\section{Properties:}

\begin{tabular}{|c|c|c|c|c|c|}
\hline $\mathbf{n}$ & $\mathbf{x}$ & $\mathbf{y}$ & $\mathbf{z}$ & $\mathbf{A}$ & $\mathbf{P}$ \\
\hline 1 & 12 & 5 & 13 & 30 & 30 \\
\hline 2 & 358 & 72 & 650 & 12888 & 1080 \\
\hline 3 & 2240 & 201 & 2249 & 225120 & 4690 \\
\hline 4 & 4794 & 392 & 4810 & 939624 & 9996 \\
\hline 5 & 8308 & 645 & 8333 & 2679330 & 17286 \\
\hline
\end{tabular}

1) $y-31 p_{n}^{5} \equiv 0(\bmod 57)$

2) $y-z+x-t_{62, n} \equiv 0(\bmod 3)$

3) $(x-15 y)(z-x)-4\left(c t_{3, n} \cdot c t_{5, n}\right)+223 n t_{6, n}-7 t_{26, n} \equiv-4(\bmod 61)$

Pattern 8:

Under our assumption

$$
\frac{A}{P}=t_{18, n}
$$

leads to the equation

$$
q(p-q)=2 n(8 n-7)
$$

The above equation is equivalent to the following two systems I and II respectively

\begin{tabular}{|c|c|}
\hline $\mathrm{p}-\mathrm{q}$ & $\mathrm{Q}$ \\
\hline $8 \mathrm{n}-7$ & $2 \mathrm{n}$ \\
\hline $2 \mathrm{n}$ & $8 \mathrm{n}-7$ \\
\hline
\end{tabular}

As in the previous case, we obtain the values of the generators $\mathrm{u}, \mathrm{v}$ and hence the corresponding sides of the Pythagorean triangle.

Case 1:

On evaluation, the values of generators satisfying system I are 


$$
p=10 n-7, q=2 n
$$

Employing (2), the corresponding Pythagorean triangle is

\section{Examples:}

$$
x(n)=40 n^{2}-28 n, y(n)=96 n^{2}-140 n+49, z(n)=104 n^{2}-140 n+49
$$

\section{Properties:}

\begin{tabular}{|c|c|c|c|c|c|}
\hline $\mathbf{n}$ & $\mathbf{x}$ & $\mathbf{y}$ & $\mathbf{z}$ & $\mathbf{A}$ & $\mathbf{P}$ \\
\hline 1 & 12 & 5 & 13 & 30 & 30 \\
\hline 2 & 104 & 153 & 185 & 7956 & 442 \\
\hline 3 & 276 & 493 & 565 & 68034 & 1334 \\
\hline 4 & 528 & 1025 & 1153 & 270600 & 2706 \\
\hline 5 & 860 & 1749 & 1949 & 752070 & 4558 \\
\hline
\end{tabular}

1) $\quad x-t_{60, n}+11 n^{2}=0$

2) $\quad n(y-2 x)-R h_{n}+t_{46, n}+26 n^{2}=7$

3) $\quad(z-y) x-z+18 t_{26, n}+141 g_{n}+190=0$

Case 2:

On evaluation, the values of generators satisfying system I are

$$
p=10 n-7, q=2 n
$$

Employing (2), the corresponding Pythagorean triangle is

$$
x(n)=160 n^{2}-252 n+98, y(n)=36 n^{2}-28 n, z(n)=164 n^{2}-252 n+98
$$

\section{Examples:}

\section{Properties:}

\begin{tabular}{|c|c|c|c|c|c|}
\hline $\mathbf{n}$ & $\mathbf{x}$ & $\mathbf{y}$ & $\mathbf{z}$ & $\mathbf{A}$ & $\mathbf{P}$ \\
\hline 1 & 6 & 8 & 10 & 24 & 24 \\
\hline 2 & 234 & 88 & 250 & 10296 & 572 \\
\hline 3 & 782 & 240 & 818 & 93840 & 1840 \\
\hline 4 & 1650 & 464 & 1714 & 382800 & 3828 \\
\hline 5 & 2838 & 760 & 2938 & 1078440 & 6536 \\
\hline
\end{tabular}

$$
\begin{aligned}
& \text { 1) } \frac{(z-x)(2 n+1)}{2}=c t_{23, n}+t_{11, n}+1 \\
& \text { 2) } 5 x-2 y-z=76 g_{n}-4 t_{50, n}-169 \\
& \text { 3) }(x-4 y)(n-1)-2\left(t_{17, n}+t_{3, n}\right)+80 g_{n}+174=0
\end{aligned}
$$

\section{Pattern 9:}

Under our assumption

$$
\frac{A}{P}=t_{19, n}
$$

leads to the equation

$$
q(p-q)=n(19 n-17)
$$

The above equation is equivalent to the following two systems I and II respectively

\begin{tabular}{|c|c|}
\hline $\mathrm{p}-\mathrm{q}$ & $\mathrm{Q}$ \\
\hline $19 \mathrm{n}-17$ & $\mathrm{~N}$ \\
\hline $\mathrm{n}$ & $19 \mathrm{n}-17$ \\
\hline
\end{tabular}

As in the previous case, we obtain the values of the generators $\mathrm{u}, \mathrm{v}$ and hence the corresponding sides of the Pythagorean triangle.

Case 1:

On evaluation, the values of generators satisfying system I are

$$
p=20 n-17, q=n
$$

Employing (2), the corresponding Pythagorean triangle is 


$$
x(n)=40 n^{2}-34 n, y(n)=399 n^{2}-680 n+289, z(n)=410 n^{2}-680 n+289
$$

\section{Examples:}

\section{Properties:}

\begin{tabular}{|c|c|c|c|c|c|}
\hline $\mathbf{n}$ & $\mathbf{x}$ & $\mathbf{y}$ & $\mathbf{z}$ & $\mathbf{A}$ & $\mathbf{P}$ \\
\hline 1 & 6 & 8 & 10 & 24 & 24 \\
\hline 2 & 92 & 525 & 533 & 24150 & 1150 \\
\hline 3 & 258 & 1840 & 1858 & 237360 & 3956 \\
\hline 4 & 504 & 3953 & 3958 & 996156 & 8442 \\
\hline 5 & 830 & 6864 & 6914 & 2848560 & 14608 \\
\hline
\end{tabular}

$$
\begin{array}{ll}
\text { 1) } & (z-y)\left(2^{n}\right)=j a l_{2 n}+3 j a_{2 n} \\
\text { 2) } & x-2 t_{42, n} \equiv 0(\bmod 4) \\
\text { 3) } & (z-y)(2 n+1)-2\left(t_{3, n+1}+c t_{3, n}-2\right)+g_{n}=0
\end{array}
$$

Case 2:

On evaluation, the values of generators satisfying system I are

$$
p=20 n-17, q=19 n-17
$$

Employing (2), the corresponding Pythagorean triangle is

\section{Examples:}

$$
x(n)=760 n^{2}-1326 n+578, y(n)=39 n^{2}-34 n, z(n)=761 n^{2}-1326 n+578
$$

\begin{tabular}{|c|c|c|c|c|c|}
\hline $\mathbf{n}$ & $\mathbf{x}$ & $\mathbf{y}$ & $\mathbf{z}$ & $\mathbf{A}$ & $\mathbf{P}$ \\
\hline 1 & 12 & 5 & 13 & 30 & 30 \\
\hline 2 & 966 & 88 & 970 & 42504 & 2024 \\
\hline 3 & 3440 & 249 & 3449 & 428280 & 7138 \\
\hline 4 & 7434 & 488 & 7450 & 1813896 & 15372 \\
\hline 5 & 12948 & 805 & 12973 & 5211570 & 26726 \\
\hline
\end{tabular}

\section{Properties:}

$$
\begin{aligned}
& \text { 1) } y\left(2^{n}\right)=28 \text { car } 1_{\mathrm{n}}+11 k y_{n}+39 \\
& \text { 2) } 20 y-z-2 t_{21, n}+274 g_{n}+852=0 \\
& \text { 3) } 39(z-x)-y \equiv 0(\bmod 34)
\end{aligned}
$$

\section{Pattern 10:}

Under our assumption

leads to the equation

$$
\frac{A}{P}=t_{20, n}
$$

$$
q(p-q)=2 n(5 n-4)
$$

The above equation is equivalent to the following two systems I and II respectively

\begin{tabular}{|c|c|}
\hline $\mathrm{p}-\mathrm{q}$ & $\mathrm{Q}$ \\
\hline $5 \mathrm{n}-4$ & $2 \mathrm{n}$ \\
\hline $2 \mathrm{n}$ & $5 \mathrm{n}-4$ \\
\hline
\end{tabular}

As in the previous case, we obtain the values of the generators $\mathrm{u}, \mathrm{v}$ and hence the corresponding sides of the Pythagorean triangle.

Case 1:

On evaluation, the values of generators satisfying system I are

$$
p=7 n-4, q=2 n
$$

Employing (2), the corresponding Pythagorean triangle is

$x(n)=28 n^{2}-16 n, y(n)=45 n^{2}-56 n+16, z(n)=53 n^{2}-56 n+16$ 


\section{Examples:}

\begin{tabular}{|c|c|c|c|c|c|}
\hline $\mathbf{n}$ & $\mathbf{x}$ & $\mathbf{y}$ & $\mathbf{z}$ & $\mathbf{A}$ & $\mathbf{P}$ \\
\hline 1 & 12 & 5 & 13 & 30 & 30 \\
\hline 2 & 80 & 84 & 116 & 3360 & 280 \\
\hline 3 & 204 & 253 & 325 & 25806 & 782 \\
\hline 4 & 384 & 512 & 640 & 98304 & 1500 \\
\hline 5 & 620 & 861 & 1061 & 266910 & 2542 \\
\hline
\end{tabular}

\section{Properties:}

1) $(z-y) x+245 c p_{n}^{6}=t_{66, n^{2}}+42 p_{n}^{5}$

2) $3 y-2 x-z-t_{54, n} \equiv 32(\bmod 55)$

3) $\frac{((2 x-z)+y)(n-1)+49 g_{n}-28}{t_{3, n+1}+t_{17, n}}$ is a nasty number

Case 2:

On evaluation, the values of generators satisfying system II are

$$
p=7 n-4, q=5 n-4
$$

Employing (2), the corresponding Pythagorean triangle is

$$
x(n)=70 n^{2}-96 n+32, y(n)=24 n^{2}-16 n, z(n)=74 n^{2}-96 n+32
$$

\section{Examples:}

\begin{tabular}{|c|c|c|c|c|c|}
\hline $\mathbf{n}$ & $\mathbf{x}$ & $\mathbf{y}$ & $\mathbf{z}$ & $\mathbf{A}$ & $\mathbf{P}$ \\
\hline 1 & 6 & 8 & 10 & 24 & 24 \\
\hline 2 & 120 & 64 & 136 & 3840 & 320 \\
\hline 3 & 374 & 168 & 410 & 31416 & 952 \\
\hline 4 & 768 & 320 & 832 & 122880 & 1920 \\
\hline 5 & 1302 & 520 & 1402 & 338520 & 3224 \\
\hline
\end{tabular}

\section{Properties:}

1) $x+z-6 y+48 g_{n}=16$

2) $z-x=2 t_{3,2 n+1}$

3) $\quad z-3 y-c t_{4, n} \equiv 31(\bmod 50)$

\section{Conclusion}

One may search for other patterns of Pythagorean triangles under consideration.

\section{References}

[1]. Albert H.Beiler, Recreations in the Theory of Numbers, (Dover Publications, New York, 1963).

[2]. Bhatia B.L., and Supriya Mohanty, Nasty numbers and their characterizations, (Mathematical Education, P.34-37, July - Sep 1985).

[3]. Dickson L.E., History of the Theory of number, (Chelesa publishing Company, New York, Vol II, 1952).

[4]. Malik S.B., Basic Number theory, (Vikas Publishing house pvt Ltd., New Delhi, 1998).

[5]. Mordell L.J., Diophantine equations, (Academic press, New York, 1969).

[6]. Ivan Niven, Zuckermann, Herbert.S, and Montgomery, Hugh.L, An introduction to the Theory of Numbers, (John Wiley and Sons, Inc, New York, 2004).

[7]. Gopalan M.A., and Devibala.S., Pythagorean Triangle: A Tressure house, proceeding of the KMA national seminar on Algebra, Number theory and applications to coding and Cryptanalysis, Little Flower College, Guruvayur, 2004, Pp.16-18.

[8]. Gopalan M.A., and Anbuselvi.R., A Special Pythagorean Triangle, Acta Ciencia Indica, VolXXXIM, No.1, 2005, Pp.53.

[9]. Gopalan M.A., and Devibala.S., On a Pythagorean Triangle Problem, Acta Ciencia indica, Vol.XXXIIM, No.4, 2006 , Pp.1451.

[10]. Gopalan M.A., and Gnanam.A, A Special Pythagorean Triangle, Acta Ciencia Indica, VolXXXIII M, No.4, 2007, Pp. 1435.

[11]. Gopalan M.A., and Leelavathi.S., Pythagorean triangle with 2 Area/Perimeter as a cubic integer, Bulletin of Pure and Applied Sciences, Vol.26E, No.2, 2007, Pp.197-200.

[12]. Gopalan M.A., and Sriram.S., Pythagorean triangle with Area/ Perimeter as difference of two squares, Impact journal of Science and Technology, Vol.2, No.3, 2008, Pp.159-167.

[13]. Gopalan M.A., and Janaki G., Pythagorean trainagle with Area/Perimeter as a special polygonal number, Bulletin of Pure and Applied Sciences, Vol.27E, No.2, 2008, Pp. 393-402.

[14]. Gopalan M.A., and Leelavathi S., Pythagorean triangle with Area/Perimeter as a Square integer, International journal of Mathematics, Computer Sciences and Information Technology, Vol.1, No.2, July- Dec, 2008, Pp.199-204.

[15]. Gopalan M.A., and Vijayasankar.A., Observations on a Pythagorean problem, Acta Ciencia Indica, Vol-XXXVIM, No.4, 2010, Pp. 517. 